\title{
Accessibility Planning for Higher Education Campuses in India: A Contextual Approach to Universal Design
}

\author{
Gaurav RAHEJA ${ }^{\mathrm{a} 1}$ and Amit HAJELA ${ }^{\mathrm{a}, \mathrm{b}}$ \\ a Department of Architecture \& Planning, Indian Institute of Technology (IIT) Roorkee \\ ${ }^{\mathrm{b}}$ School of Planning \& Architecture, New Delhi
}

\begin{abstract}
Accessibility in higher education campuses of India paves way for inclusion.This paper shares perspectives from three diverse campuses from India and highlights the accessibility paradigms in their respective contexts. It further elaborates the contextual measures of accessibility and universal design from these examples with larger focus on physical attributes of accessibility. Challenges of historic and mixed use campus alongwith high ecological footprint pose distinct perspectives to accessible built environments in higher education. Comparative understanding of accessibility through structured metrics and mapping with Universal Design goals leads to development of a framework to assess and guide universal design approach in higher education in similar contexts. It argues that Universal design approach requires a contextual interpretation for contexts like these and may reflect new interpretations to existing theories. New Education Policy by the Government of India and Covid '19 as pandemic have furthered the need and understanding of accessibility in higher education with some degree of universaliaton and some degree of contextualization.
\end{abstract}

Keywords. Accessibility Planning, Higher Education, Universal Design, India, Inclusion

\section{Introduction \& Background}

Higher education in India spreads across disciplines and cultures spanning regional, climatic, socio economic and cultural diversity. More so, the contemporary context of surge in educational paradigms and awareness of inclusion and access amidst the release of New Education Policy (NEP), 2020 [3] forms a considerable momentum to adopt Universal Design approach seamlessly. National institutes or universities predominantly offer this dimension of it's constitution all across in areas of technology, management, design, medicine and others. Accomodation and inclusion of persons with disabilities in higher education got initiated mostly after passing of the Persons with Disabilities (PwD) Act in 1995 which got further strengthened in the year 2016 by a new Rights of Persons with Disabilities (RPwD) Act and now a sector specific policy i.e NEP, 2020 paves way for further integration of education with inclusivity. Accessibility in higher education in India remains a subject requiring greater professional attention than ever before for multiple reasons including that of legal compliance. More importantly, India has a large

${ }^{1}$ Corresponding author. 
number of existing campuses and universities and a good number is emerging for future of higher education both by the government and the private sector. It is apt to realize and promote the application of universal design (UD) in these contexts of education so as to include a larger section of youth in the realms of higher education. This paper presents three selected case studies of prestigious national campuses of professional education with distinct historical precedents and scales reflecting the broad approaches of accessibility being employed. The campus administration and faculty remain sensitive to the idea of accommodation of students with special needs but require greater support and specific inputs from physical and technological infrastructure to be able to respond to inclusion.

Accessibility planning for higher education campuses remains an area for significant interventions in the landscape of Indian education systems. Evolving from the charity or medical model approach to the social model requires transformations in infrastructure, information and services of any education system. The Rights of Persons with Disabilities Act, 2016 as a recent development strengthens the concept of universal design and non discrimination ahead from the earlier act of 1995. Besides makes provisions for three to four percent reservation in educational institutions for students with disabilities, it also specifies a timeline of 5 years from the passing of the act for all infrastructures to become accessible.

Also, the classification of types of disabilities has increased from seven in the earlier act to twenty one in the recent one. In light of positive developments of enforcement and legal provisions for access, ground implementation of accessibility needs to be pushed beyond mere code compliance to achieve an ideal of inclusion in education and other sectors in the twenty first century.

The National Building Code (NBC) released in the year 2016 forms yet another instrument of implementing accessibility in a mandatory form than a recommendatory one. It proposes the idea of execution of accessibility between external and internal environments implying a holistic synergy and seamless access throughout a campus or institutional experience. It's important to understand that access in higher education or any other form begins with mobility, transforms into learning and impacts social participation. Together, it integrates into inclusion through the subtle transitions between infrastructure and policy frameworks. UD no longer remains an idea of removing barriers but of creating positive spaces with seamless integration of diversity. Translating this in reality poses distinct challenges in existing campuses while the new ones can address this in much more impactful ways through infrastructure design and policies.

India and the Indian context offers a fairly pluralistic dimension of human diversity which poses both uniqueness and challenges towards access and inclusion in education and beyond. However, in order to accommodate individuals with disabilities, educational campuses need to have a holistic strategy towards a seamless integration of diverse forms of students. Through shared experiences and live case study approach this paper highlights diverse perspectives of incorporating accessibility in higher education environments. It further emphasizes how the understanding of universal design approach can enhance education not merely as built or physical experience but as knowledge and learning experience on campuses. 


\section{Universal Design India and Indian education scenarios}

Universal Design, even though popular through the terms of accessibility, inclusion and barrier free approaches in education sector needs a contextual view in India and the global south. Even without the term in it's literal sense, the ideology of universal design is prevalent in it's partial forms of education and daily living with immense scope to further the same. Besides the goals of universal design from it's roots in the United States, the Universal Design India Principles advocate two critical dimensions viz. economic affordance and cultural appropriateness. Indian education systems though have a large scale and an immensely high footfalls still requires to exert the true sense of inclusion through it's infrastructure.

Recent introduction of the New Education Policy by the Government of India in 2020 brings a focus on inclusive education and interdisciplinarity which perhaps could enable the idea of it's reach and connect with the diverse forms of youth in the country. Universal design as a base theory and approach requires a framework to dovetail into the Indian educational think tank for all levels including primary to higher education. This implies a strategic approach towards accessibility in education through physical infrastructure, educational content delivery and allied services and regulations related to it. The paramount principles of flexibility in design, enabling learning through distance and digital modes, assistive technologies support, policies for conducting exams in accessible formats, etc. shall go a longway in transforming the social environments on educational campuses.

Indian reservation policy for students with disabilities remains one of the institutional ways to bring in access to education, even though the true impact of such policies would require a multi pronged strategy towards developing infrastructure and educational content and methodologies. Covid'19 pandemic brought in sudden impacts to educational paradigms and continues to draw both lines of inclusion and exclusion into contemporary higher education. Digital and online modes of education emerged to be the most widely employed modes of education implying diverse perspectives to adaptations and delivery. In some cases, it made education more flexible with greater choice using new forms of softwares with digital accessibility, however in some cases the struggle remained with basic infrastructure challenges of economic affordances of digital devices and internet connectivity as new perspectives for those who couldn't afford it.

Universal Design as an approach and philosophy remains a continuous lens to guide transformative strategies in higher education and new forms of delivery models ensuring access to all. It needs to evolve with continued efforts, understanding and innovation to be able to sustain the idea of inclusion for all in higher education. On a positive note, such awareness is emerging in many Indian institutions and centers of higher learning alongwith regulatory bodies to ensure access through it's mandate. Regulatory bodies like All India Council for Technical Education (AICTE) and University Grants Commission (UGC) have formally initiated steps to evolve frameworks for introducing accessibility into higher education both as academic courses and in the spirit of delivering inclusive service to all. 


\section{Methods}

\subsection{Study context}

Three distinct educational campuses of large scale and high repute and ranking were chosen for this study engaged in professional education from Indian conext. The reason and criteria for their choice was diversity of context, live experience of authors in all campuses alongwith access to best practices and interactions with stakeholders. Comparative reviews of these campuses, feedbacks from students with disabilities, access audit processes and grounded engagement as consultants in accessibility provisions of campuses together form the components of a structured methodological approach. Several learnings emerge from experiential trace studies, walk and talk audits from these campuses sampled for higher education in technical, management, medical domains.

\subsection{Participants}

The participants included diverse category of stakeholders including students with disabilities. Qualitative interviews were conducted to understand their personal insights into accessing campus from multiple perspectives. The authors themselves were involved in trace walks on these campuses to evaluate the physical accessibility of infrastructures.

\subsection{Data collection and analysis}

Comparative reviews of educational campuses, feedbacks from students with disabilities, access audit processes and grounded engagement as consultants in accessibility provisions of campuses together form the components of a structured methodological approach.

\section{Accessibility Review and Case Findings}

Campus infrastructures of the chosen campuses of IIT Roorkee, IIM Bangalore and Jamia Hamdard University have been analysed through perspectives of accessibility planning, spatial quality and contextual issues from Indian academic systems. The study brings out a structured assessment of accessibility through building standards compliance and also through qualitative perspectives of campus planning. It highlights the challenges and outcomes of retrofitting for accessibility on a historic campus of IIT Roorkee (174 years old) alongwith insights into campus of IIM Bangalore alongwith developing a new campus of Jamia Hamdard university incorporating universal design strategies in it's urban design. The findings of this study highlight and advance the understanding of universal design in master planning and detailing educational campus infrastructures for inclusion. 


\subsection{Accessibility on IIT Roorkee campus}

IIT Roorkee is a historic campus of higher education set up in the erstwhile British rule, almost 174 years ago in the year 1847. The most prominent historic and iconic administrative building today known as James Thomason Building forms part of the campus heritage. Spread over 365 acres, the campus grew heterogeneously in the post independent India and with mixed use zoning, predominantly old structures and an arterial street radially connecting most campus zones from academic to residential ones. By generic understanding of a campus plan, it remains an extremely walkable and a free wheeling campus meaning bicycles and wheelchairs can inclusively move around barring barriers of level coordination, etc. However, the challenges in physical aspects of getting around the campus begin to matter in terms of distances, time and weather conditions amidst a busy academic time schedule. Students with locomotor disabilities have expressed some of these challenges and begin to reflect into strategies to mitigate them.

The campus administration has been consistently making it's own physical infrastructure accessible and respond holistically to the needs of students with disabilities. With a population of over 100 students with disabilities on campus, the idea of inclusion starts reflecting in some measure through greater human and infrastructure sensitivities [2]. Accessibility as both retrofitting strategy for existing built forms and as an embedded principle for new constructions remains widely followed. Universal design as a theory needs reinterpretation and contextualization for various aspects of implementation. One of the key challenges is to sensitise working groups about the various academic, mobility, recreational or spatial needs of living on this campus while the other to constantly undertake accessibility in educational content delivery systems.

As a vision for accessibility on IIT Roorkee campus, it is intended to gradually mould the campus built environment from a barrier free concept to an inclusive experience guided by universal design and greater accommodations for individuals with disabilities. Predominantly the campus initiatives have been instrumental in retrofitting physical infrastructure with adequate ramps (more than 25) and accessible entrances of most academic and administrative built forms, providing accessible washrooms and equip elevators in most of the existing infrastructure in the past decade.

An inclusive experience of a type that would facilitate an academic, social, recreational and professional integration of persons with disabilities (like mobility impaired, wheelchair users, low sighted, blind, hearing impaired, etc.) alongwith able bodied participants in its spaces like departments, administrative offices, outdoor interactive areas including sports and informal campus zones, etc. While our initial focus is to create barrier free access (at ground floor level) in campus buildings, it requires to remove a series of physical barriers that may otherwise restrict access to our built environment spaces. The campus environment at that stage would not only have physical access to the widest possible extent but also would like to support the special needs for all categories of disabilities through its infrastructure facilities.

The physical barriers like levels, steps etc. at the entrances of buildings can be counteracted by adding features like ramps (with adequate slopes) to facilitate needs of the mobility impaired. Support hand rails and guiding blocks with tactile impressions on 
the walking surfaces further enhance the mobility support to users with low vision or blindness. Further, inaccessible toilets were transformed into accessible ones bywidening doorways with a lever handle operated door, accessible pathways and appropriately modifying the internal configuration of the w.c. for greater comfort in itsuse while transferring from a wheelchair to the seat. While these features aim to cater to a wide range of human needs, they shall always continue to be design conveniences for the able bodied in the campus built environment.

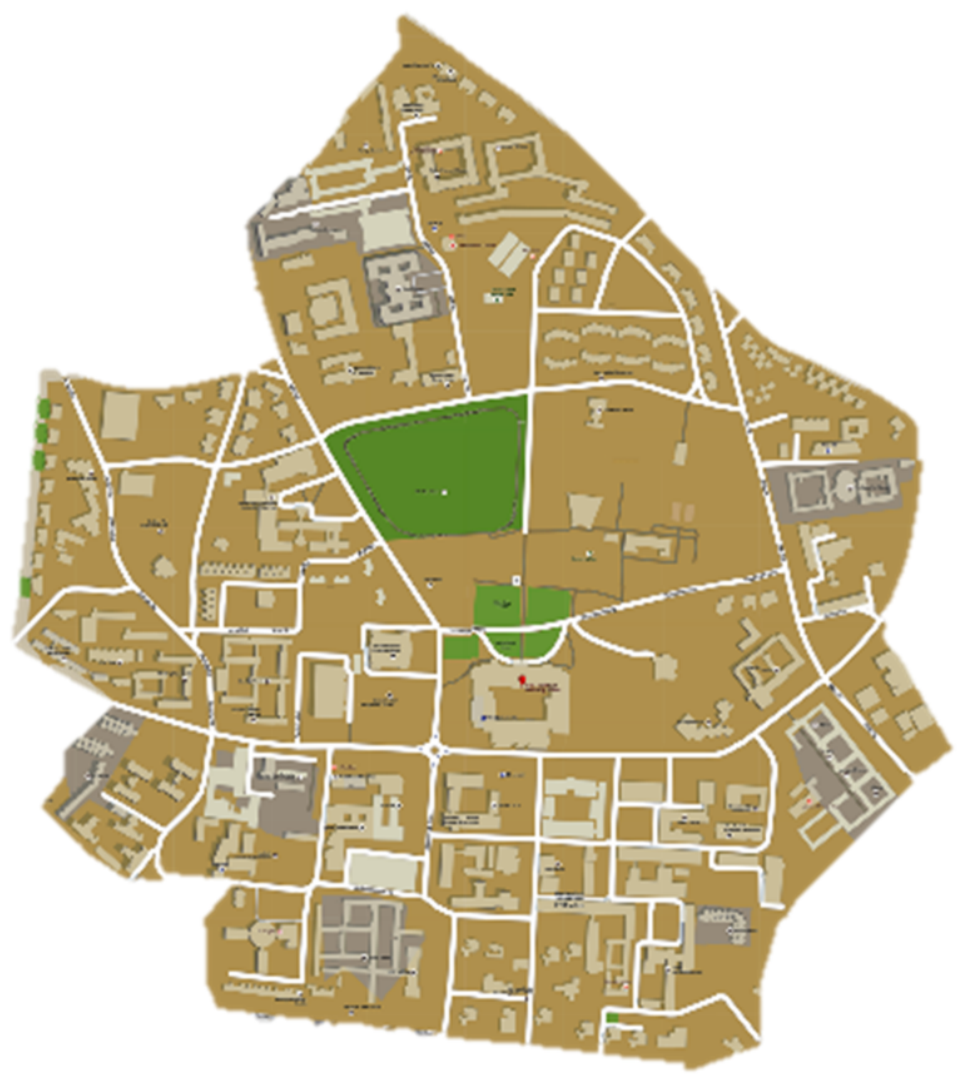

Figure 1 Campus Layout Plan, IIT Roorkee (Source: iitr.ac.in)

Grounded surveys have been conducted amongst students with disabilities to understand their special needs for accommodation and experiences. Various initiatives including provision of scribes during examination alongwith extra time provisions and additional support for content learning remain part of ongoing policy for academic inclusion of students with disabilities. The students through qualitative interviews suggested areas of further intervention for access including the sports and gym access alongwith hostel room allocation preferences. IITR campus has also been making provisions for personal assistive technologies for students with disabilities and recently founded Team Inclusion under the Chair of Inclusion and Accessibility Services on it's campus. 


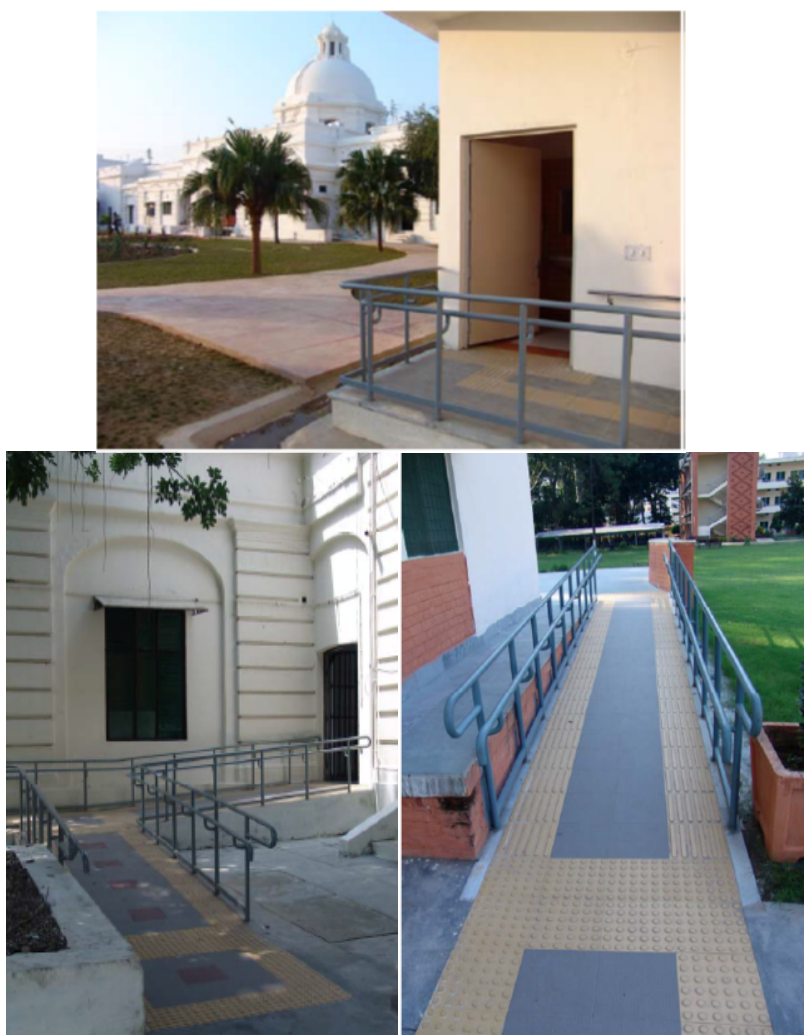

Figure 2 Retrofitting for accessibility showing access ramps and pathways to existing built environments at IIT Roorkee (Source: Authors)

The above figure illustrates the development of accessible infrastructure through retrofitting all across the campus in phases. It would be vital to note that being public funded institution, provisions for accessibility require sensitization and awareness of the infrastructure planning sections of administration which could then strategise a phase wise plan to retrofit old campuses with accessible infrastructure. Universal Design in terms of accommodation for size and space for diverse population groups alongwith equitable use continue to be addressed in these historic yet progressive campuses.

\subsection{Accessibility on IIM Bangalore campus}

The campus at IIM Bangalore was designed by Pritzker prize winning architect B V Doshi on the ideals and philosophy of contextual responsiveness incorporating climate sensitivity. The campus so planned with a loose integration of a grid which punctuates itself with enclosed, semi open and open to sky spaces amidst a thick green cover. The campus is usually celebrated as a spatial vocabulary of built forms nestling in nature. 
"The architecture of the IIM resists the clear and precise image. Over time and through repetition, one can grasp the arrangement of the plan and the sequence of spaces that flank the corridors but by negating formal clarity, the building gives preference to the experience of traversing through its in-between spaces. The spatial experience is heightened by the frequent changes of scale and the occasional breaking of the form of the corridor. The allowance of the landscape to intersperse with the built form enables the building to create soft, loosely defined edges."

-Architect B V Doshi

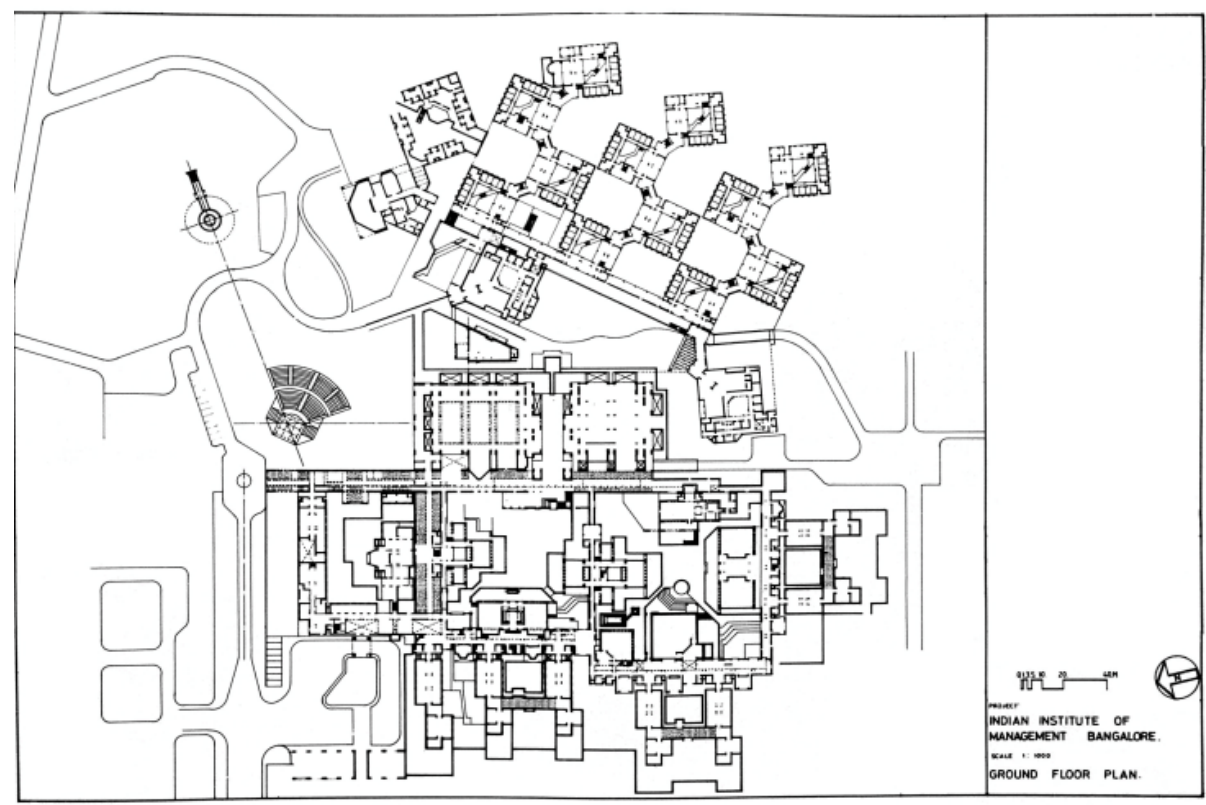

Figure 3 Campus Layout Plan, IIM Bangalore (Source: iimb.ac.in)

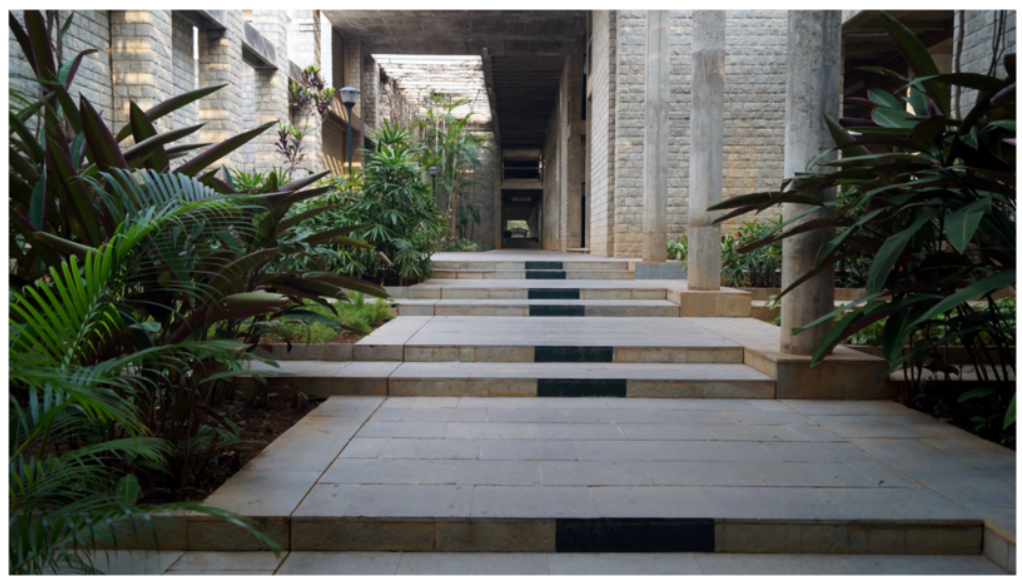

Figure 4 Campus Interconnecting Pathways, IIM Bangalore (Source: iimb.ac.in) 
With a blend of ecology and local stone, the campus forms a background of grey and greens keeping a earthy profile amidst it's academic and research profile. Known for it's unique identity of architecture, accessibility could be critiqued for the range of stepped levels that this campus has with minimal grab rail support. However, the overall planning suggests an integrated network of walkable streets and pathways. The courtyard planning as part of a climatic response to the architecture also provides a nice daylight experience for individuals to walk in. The stepped levels also provide for multipurpose activities of academic or informal discussions amongst peers and others.

Built as a campus in late $70 \mathrm{~s}$ and early $80 \mathrm{~s}$, it offers yet another contextual experience of understanding universal design in an Indian context, wherein climatic responsiveness and spatial functioning with master planning for walkability could make it largely equitable and usable. However, introduction of other accessibility features got initiated much later, which now through connected ramps, intermediate lifts and other retrofitting strategies continues to be carried out on campus to support students with disabilities. Campus expansions with intermittent new additions however are conscious of accessibility provisions including the guest house and the

The Office of Students with Disaiblities on IIMB campus has initiated some exemplary steps to support the academic and assistive technology needs of diverse students with disabilities including sensory and locomotor challenges. Office of Disability Studies on IIM Bangalore campus continuously strives towards taking initiatives towards providing inclusive support for their special needs as part of academic or campus living concerns. IIM Bangalore remains one of the leading institutions for a campus accommodation policy and has a paradigm for the same.

IIMB remains one of the best known sensitive designs practices from Indian context. Accessibility as a concept of campus planning remains one of the highlights from the ground understanding of trace studies with intermittent features of retrofit access on campus. Tactile guiding systems were not commonly employed as part of physical infrastructure though the campus has empowered the students with visual impairments through technological interface during the Covid'19 times and has showcased exemplary approaches to digital inclusion in higher education.

\subsection{Accessibility initiatives on Jamia Hamdard campus}

Jamia Hamdard University (JHU) was established in the year 1962 in New Delhi by the then Prime Minister of India funded by a private Trust foundation. Like the other two cases, this university continues to be a university of excellence in the education of pharmacy primarily. However, it has grown into a full fledged university with engineering, basic sciences and medicine as it's other domains of academic expansion.

Considering this too as a development from the 1960s, the university evolved itself with a sizeable infrastructure for long. However, since over a decade amidst the redevelopment and expansion projects of campus. It is evident to note that with the rise in awareness and sensitivities towards inclusion of students with disabilities in higher education, the translation into the architectural features including streets and other external environments and design features on the inside building features has started growing. Tactile paver blocks and level management remain two key criteria in 
enhancing accessibility across the campus. Figure 5 below highlights the mobility master plan with segregation between pedestrian and vechicular mobility. Keeping a pedestrian focus enhances accessibility as an overall concept besides making it more sustainable and inclusive.

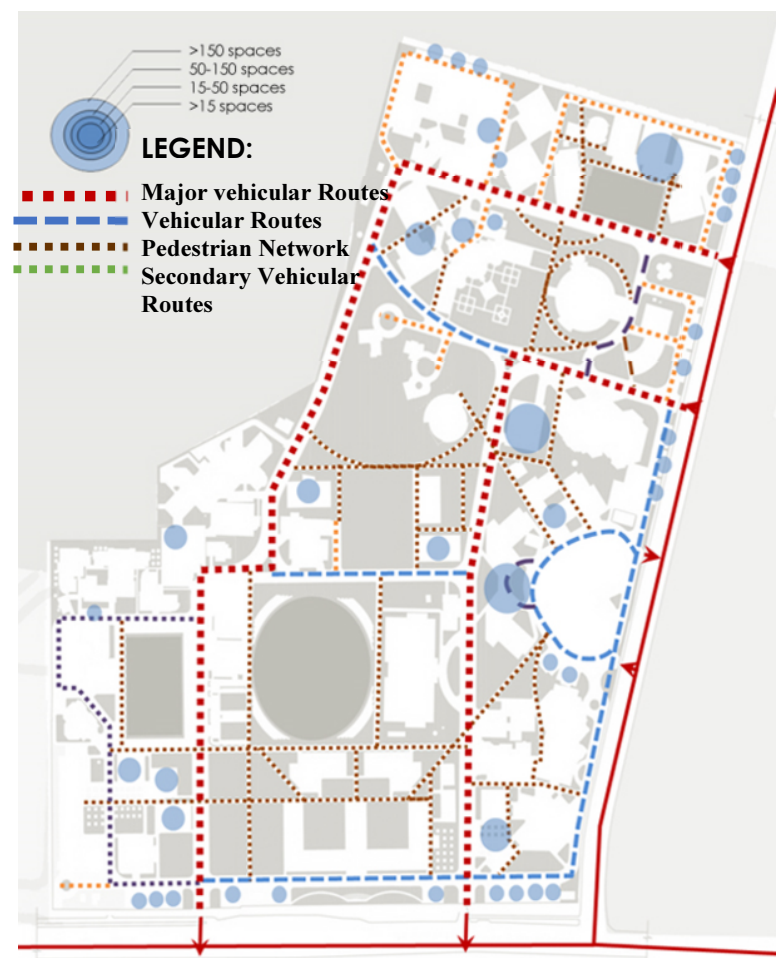

Figure 5 Movement pattern, Campus Redevelopment Plan, Jamia Hamdard University (Source: Authors)

Accessibility remains an integral element for the master planning activity as proposed for 2030 with integral walkways, table top crossing within the university. Besides this the new lecture hall complex was designed and executed recently as a Smart Classroom cluster. The floor plate design takes care to accommodate a wide range of accessible classrooms on the ground floor itself, while integrating accessibility services as part of the overall building complex. Accessible washrooms were integrated in new residential forms of hostel rooms and central corridors were connected with upper levels through ramps as per harmonized guidelines published by the Government of India.

JHU too has a mix of old and new infrastructure which is typical for most Indian academic institutions of higher learning existing from post Independent times. Universal Design approach has in the recent years started gaining acceptance for newer establishments, however the barrier free approach remains a predominant method of approaching retrofitting. Students with disabilities broadly under the reservation. Pedestrian plaza (as shown in Figure 6) designed for the campus integrated the concept of inclusion through physical element designs in the external environment of the campus. 


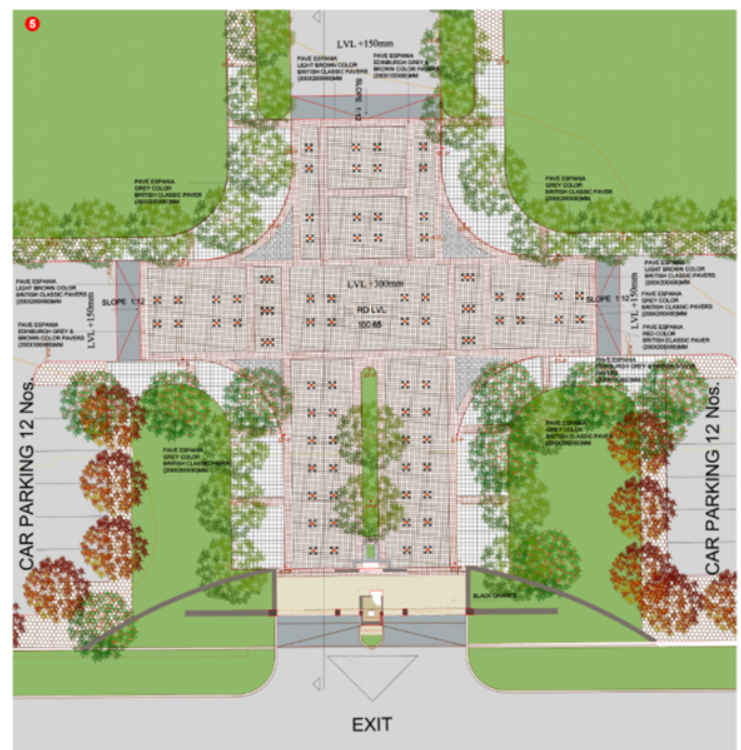

Figure 6 Redesign of Pedestrian Plaza as part of Master Planning, Jamia Hamdard University (Source: Authors)

Accessibility measures in master planning and physical forms have further accelerated demand and sensitivity amongst campus communities. This leads not only to better awareness of these features but helps impact the social cultures of inclusion in higher education campuses. The connect with city accessibility and larger attributes still remains a zone for further works.

\section{Discussions and Comparative Analysis}

Campus infrastructures of the chosen campuses of IIT Roorkee, IIM Bangalore and Jamia Hamdard University have been analysed through perspectives of accessibility planning, spatial quality and contextual issues from Indian academic systems. The study brings out a parametric assessment of accessibility through building standards compliance and also through qualitative perspectives of campus planning. It highlights the challenges and outcomes of retrofitting for accessibility on a historic campus of IIT Roorkee (174 years old) alongwith insights into developing a new campus of Jamia Hamdard university incorporating universal design strategies in it's urban design. The findings of this study highlight and advance the understanding of universal design in master planning and detailing educational campus infrastructures for inclusion. 
Table 2. Accessibility Framework for Higher Education Campuses (Source : Author)

\begin{tabular}{|c|c|c|c|}
\hline \multirow[t]{2}{*}{ S.No. } & \multicolumn{2}{|c|}{ Accessibility Framework for Educational Campuses } & \multirow{2}{*}{$\begin{array}{ll}\text { Related } & \text { UD } \\
\text { Goals [ 1 ] } & \end{array}$} \\
\hline & Thematic Clusters & Elements / Attributes & \\
\hline 1 & Master Planning & $\begin{array}{l}\text { Zoning, Landmarks, Academic-Non } \\
\text { Academic areas, Spatial Grids, } \\
\text { Placemaking }\end{array}$ & $\begin{array}{l}\text { Goal no. } 2 \\
\text { (Comfort) and } \\
5 \text { (Wellness) }\end{array}$ \\
\hline 2 & Mobility \& Circulation & $\begin{array}{l}\text { Distance, Networks \& } \\
\text { Interconnections, Parking and } \\
\text { Pathways, Internal movement, } \\
\text { Vertical circulation }\end{array}$ & $\begin{array}{l}\text { Goal no. } 3 \\
\text { (Awareness) and } \\
4 \\
\text { (Understanding) }\end{array}$ \\
\hline 3 & Core Academic Infrastructure & $\begin{array}{l}\text { Classrooms, Laboratories, Seminar } \\
\text { Rooms, Conference Rooms, } \\
\text { Washrooms, Drinking water facility, } \\
\text { Reception areas, Entrances }\end{array}$ & $\begin{array}{l}\text { Goal no. } 6 \\
\text { (Social } \\
\text { Integration) and } \\
8 \text { (Cultural } \\
\text { Appropriateness) }\end{array}$ \\
\hline 4 & Spatial Quality & $\begin{array}{l}\text { Reachability, Usability, Flexibility, } \\
\text { Interactivity, Materiality, Scale \& } \\
\text { Proportion }\end{array}$ & $\begin{array}{l}\text { Goal no. } 7 \\
\text { (Personalization) } \\
\text { and } 8 \text { (Cultural } \\
\text { Appropriateness) }\end{array}$ \\
\hline 5 & Educational Services & $\begin{array}{l}\text { Assistive Technology, Administrative } \\
\text { services, Academic Services, } \\
\text { (Teaching and Assessment), } \\
\text { Disability Services, Technology } \\
\text { services }\end{array}$ & $\begin{array}{l}\text { Goal no. } 2 \\
\text { (Comfort) and } 7 \\
\text { (Personalization) }\end{array}$ \\
\hline 6 & Contextual Dimensions & $\begin{array}{l}\text { Culture, Climate, Materials, Policy, } \\
\text { Planning, Formal-Informal } \\
\text { activities, Economic backgrounds, } \\
\text { Location, Ownership type }\end{array}$ & $\begin{array}{l}\text { Goal no.6 } \\
\text { (Social } \\
\text { Integration) and } \\
8 \text { (Cultural } \\
\text { Appropriateness) }\end{array}$ \\
\hline
\end{tabular}

Master Planning plays a vital role in giving character of everyday life and mobility on campus. Through a choice of zoning various academic functions the three campuses reflect traces of inclusion in pockets where seamless accessibility is evident. All three campuses have undertaken master planning as an activity for future growth and improvisation of state of the art services. However, the challenges remain unique to each of them owing to their independent contexts and existing infrastructural perspectives. Accessibility remains one of the key agenda amidst other dimensions of master planning in all three contexts and efforts to achieve them through street design, new built form developments and strategies to approach informal campus spaces remain as key highlights of master planning for inclusion.

Mobility and Circulation forms one of the key concerns for students with disabilities. All three campuses reflect a pedestrian scale of development with decent walkable infrastructure not completely free from barriers, especially crossings. IIM Bangalore campus as conceived structures mobility predominantly through corridors and a mix of spaces supported by a peripheral vehicular access. The campus forces a pedestrian scale of mobility making it more humane as an experience minimizing conflict of pedestrian movement to vehicular one. JHU on the other hand offers a scale of greater vehicular movement which is restricted or supported through intersecting plazas which not only 
become measures of traffic calming but also offer a recreational informal space for interactions in outdoor environments.

Core Academic Infrastructure becomes yet another critical dimension of inclusion in higher education. Through an understanding of the campus case studies, it was found that some students with disabilities face challenges at personal level in either exerting extra hard to connect with the academic components of the education, especially conducting laboratory experiments in engineering and teaching learning attributes. Sensitivity amongst faculties surely leads to some degree of accommodation but there remains a scope in infrastructure and technologies to make the whole experience more enabling. Classroom designs with stepped seating pose a challenge to the wheelchair users especially when the space between the front stage (raised platform) and the first row of seating is slightly high. Such aspects were attributed in the new designs of classroom planning in the JHU campus.

Once part of a formal space in the academic areas, students, faculties and staff require to use other supporting services including the washrooms. Accessibility provisions in these washrooms through retrofit model pose a tremendous challenge both for infrastructure as well as for ongoing academic programmes due to lack of spaces and other plumbing issues. While at IIT Roorkee, it has been proactively possible to make such accomodations in most of it's campus buildings as a retrofitting exercise, new building projects in all three campuses are already incorporating universal design practices for their academic environments.

Spatial quality is an intangible dimension of inclusion. Any educational campus by virtue of it's spatial quality reflects an image of being human centric through a cumulative experience of mobility, interactiveness, inter connectivity, scale, landscapes, wayfinding, resting spaces, etc. Accessibility and sensitivity to spatial quality of small and large scale campus spaces leads to a more inclusive culture. IIT Roorkee has a wide range of spatial experiences on it's campus. Heterogenity and mixed use lead to availability of distinct choices. As experienced on the campuses of IIT Roorkee and IIM B individuals with disabilities are able to use the physical and spatial environment in terms

Educational services in all the three campuses are known for their quality in diverse domains of technology, management and pharamacy. Steps have been undertaken to enhance inclusivity in educational services predominantly through digital inclusion services during the Covid'19 times. However, shared practices further strengthen the model of educational deliveries across campuses. Shared learnings across institutions would be one way to exemplify best practices across institutions.

Contextual Dimensions reflect a holistic approach to a multitude factors of campus accessibility. This includes the physical aspects of site context to social context of educational environment so conceived. All the three chosen campus contexts have their distinct climatic, ecological and socio cultural contexts. More importantly also the educational programmes and cognitive orientation also shapes the contexts distinctly with independent campus cultures. The common dimensions of these contexts being a mix of old and new structures and requirements of retrofitting across a large scale to achieve physical accessibility in their respective infrastructures. The ongoing pandemic 
on the contrary shaped a collective context of adaptation to digital education formats with new forms of inclusion in educational content. Though a long term impact of this is yet to be analysed, it showed ample evidence of progressive features of accessibility being adapted at low costs by these campuses.

The above discussion and table of various accessibility attributes paves way for advancing research on accessibility assessments in higher education campuses.

\section{Concluding Remarks}

Inclusion in higher education remains a long term goal to be progressively achieved both in spirit of law and human rights alongwith a socially pressing need. This paper showcases a paradigm of accessibility in higher education from three distinct examples of Indian campuses in the domain of technical, management and medical education. It highlights the challenges and efforts being made to steer campuses towards a sustained idea of inclusion of persons with disabilities. Governmental initiatives exist yet the gaps remain at the levels of enforcement and implementation in a holistic way.

Accessiblity planning for new campuses paves way through master planning as demonstrated through the example of Jamia Hamdard University, wherein through a structured urban design process it is being ensured to have seamless connect and mobility through the campus. IIT Roorkee and IIM Bangalore suggest different contexts of retrofitting and progressive approach to inclusion.

Accessibility is the key bridge to inclusivity. While higher education campuses need to integrate efforts towards creation of more accessible campuses, primary and secondary education sectors in India shall need to steer greater efforts so that students with disabilities could further pursue education in higher education contexts. Architecturally speaking, the three campuses discussed in this paper inform about distinct approaches towards the practice of universal design. It comprehends the connect between the guiding parameters and their attributes.

Higher education paradigms of India need to raise further standards of accessibility not from a compliance perspective but from a best practice approach. The New Education Policy lays sufficient thrust on the idea of inclusion in educational environments. The ongoing pandemic situation of Covid'19 has further demonstrated opportunities of digital inclusion and some forms of disruptive innovations in educational trends.

Such efforts boost confidence amongst those with disabilities and goes a long way in promoting independent functioning and social inclusion in education environments. However, it is critical to translate that understanding while planning and creating educational infrastructure. The paper concludes by highlighting the accessibility framework and it's contextual connect with universal design theory in representative Indian contexts.

Universal Design is convincingly an inclusive and an open approach with wide range of interpretations keeping the principles of equity and accessibility sacrosanct. 
Indian perspectives of higher education are contextually evolving accessibility in their given contexts while the policies have always favoured equity as a constitutional right. Amidst ongoing pandemic times, the future of hybrid education needs to be prepared for with greater scope of inclusion and access through both technology and human support.Such forms and qualities of higher education would perhaps sustain beyond their campues too and could be accessible and inclusive to a wider range of students with or withtout disabilities.

\section{Acknowledgments}

We thank the three Institutions viz. IIT Roorkee, IIM Bangalore and Jamia Hamdard University for their kind support and the participants of our survey for their voluntary participation and valuable time.

\section{References}

[1] E.Steinfeld and J.Maisel, "Universal Design: Designing Inclusive Environments," Wiley, USA, 2012.

[2] G.Raheja, "Accessiblity Planning for IIT Roorkee campus", Unpublished report, 2009.

[3] Ministry of Human Resource Development, "New Education Policy 2020" Government of India, 2020.

[4] Rights of Persons with Disabilities Act, 2016 https://legislative.gov.in/ actsofparliamentfromtheyear/rights-persons-disabilities-act-2016

[5] www.iimb.ac.in.

[6] www.iitr.ac.in 